\title{
Shocks in direct numerical simulation of the confined three-dimensional mixing layer
}

\author{
Bert Vreman, Hans Kuerten, and Bernard Geurts \\ Department of Applied Mathematics, University of Twente, P.O. Box 217, 7500 AE Enschede, \\ The Netherlands
}

(Received 18 November 1994; accepted 18 April 1995)

\begin{abstract}
The occurrence of shocks in the confined three-dimensional turbulent mixing layer at convective Mach number 1.2 is established by means of direct numerical simulations. The shocks are generated by the turbulent motions in the flow. Consequently, they can have different shapes and orientations, while they persist for a relatively short time. Furthermore, they are created by different types of turbulent vortices. The shocks do not strongly contribute to the turbulent dissipation. Even at the time when the largest shocks occur, the fraction of the turbulent dissipation due to the shocks is less than 10\%. (c) 1995 American Institute of Physics.
\end{abstract}

In recent years, increased research effort has been directed toward the compressible turbulent mixing layer. ${ }^{1} \mathrm{Nu}-$ merical simulations of the two-dimensional compressible mixing layer have shown that shocks appear when the convective Mach number $M_{c}$ is higher than 0.7 . However, in simulations of the three-dimensional mixing layer, shocks have not been observed up to $M_{c}=1.05,{ }^{2}$ and whether they occur in three-dimensional flows at higher convective Mach numbers is still an open question. ${ }^{1}$ In this paper we report the occurrence of shocks in a direct numerical simulation (DNS) of a highly three-dimensional confined mixing layer at $M_{c}=1.2$.

The flow is investigated within a temporal framework in the domain $\left[0, L_{1}\right] \times\left[-\frac{1}{2} L_{2}, \frac{1}{2} L_{2}\right] \times\left[0, L_{3}\right]$. Periodic boundary conditions are imposed in the streamwise $\left(x_{1}\right)$ and the spanwise direction $\left(x_{3}\right)$, whereas the normal direction $\left(x_{2}\right)$ is bounded by free-slip walls. The equations are nondimensionalized with a reference length, which is half the initial vorticity thickness, and a reference density, velocity, temperature, and viscosity that equal the initial upper stream values. The Reynolds number based on these reference values equals 100. In the nondimensional description, the initial mean profile for the streamwise velocity is given by $u_{1}=\tanh x_{2}$, whereas the initial pressure is uniform and the BusemannCrocco law prescribes the temperature. Initially, a pair of equal and oblique disturbances is superimposed on the mean profiles. ${ }^{2,3}$ We use $L_{1}=39.9, L_{2}=59$, and $L_{3}=22.1$, where $L_{1}$ and $L_{3}$ correspond to wavelengths of the most unstable oblique mode from linear stability analysis.

The Navier-Stokes equations are solved with a combined numerical discretization employed, using fourth-order accurate central differences in the absence of shocks ${ }^{4}$ and the third-order accurate MUSCL-scheme in the presence of shocks. ${ }^{5}$ The flow is simulated on a uniform grid until $t=240$. From $t=0-80$ it contains $240 \times 385 \times 144$ points, whereas from $t=80$ the simulation is performed on a grid with $320 \times 513 \times 192$ points. A fourth-order accurate interpolation method is used to transfer the field at $t=80$ to the finer grid. The accuracy in the linear regime has been checked by comparisons with linear stability theory. Furthermore, additional calculations on coarser and finer grids established the accuracy of the results presented in this paper. The demand for computer resources could substantially be reduced by ex- ploiting the symmetries in the flow. Symmetries in the initial conditions are known to persist in time even after mixing transition, ${ }^{3}$ and we have verified this for the coarser grid simulations.

In the flow a transition to small scales is observed. At $t=80$ the growth of the initial perturbation has saturated, nonlinear effects have set in and a $\Lambda$-vortex structure has developed. When time proceeds, additional vortices are formed away from the central layer. These vortices break down into smaller vortices, and thus the flow undergoes a transition to small-scale turbulence, similar to the flow at a convective Mach number of $0.8 .^{3}$ During this process the momentum thickness rapidly grows and is approximately linear in time. At several times shock waves are observed.

The Reynolds number (100) used in the simulation should not be much lower for shocks to occur. In a simulation with a lower Reynolds number (50) the mixing transition did not occur, and no shocks were formed either. Thus, the occurrence of shock waves in the three-dimensional mixing layer appears to require a mixing transition to small scales. The flow is well resolved, since the falloff in the two-dimensional energy spectrum was about 12 orders of magnitude at most times throughout the simulation. Only at times when shocks were present a falloff of eight orders of magnitude was observed. Although these observations confirm that the resolution is sufficient, the calculations should not be performed on much coarser grids. For example, the shocks at $t=182$ and from $t=200$ were not observed in a calculation performed on a $160 \times 256 \times 96$ grid.

The flow contains shock waves in three distinct periods: $t=121-123, t=181-183$, and $t=199-225$. Since a shock corresponds to a large pressure difference in the direction perpendicular to the shock, it can be detected by examining the flow field at times where at least one of the components of $\nabla p$ shows a high peak. Furthermore, since shocks lead to a strong compression of the fluid, the dilatation $\nabla \cdot \mathbf{u}$ attains large negative values within a shock.

First, we investigate the shocks in the first period ( $t$ $=121-123$ ) and determine their origin. Maximum values of the pressure gradient occur in the center plane $x_{2}=0$. Figure 1(a) shows contour lines of the pressure in this plane at $t=122$, and four shocks are observed. Since there are two symmetries in the center plane, it is sufficient to consider the 

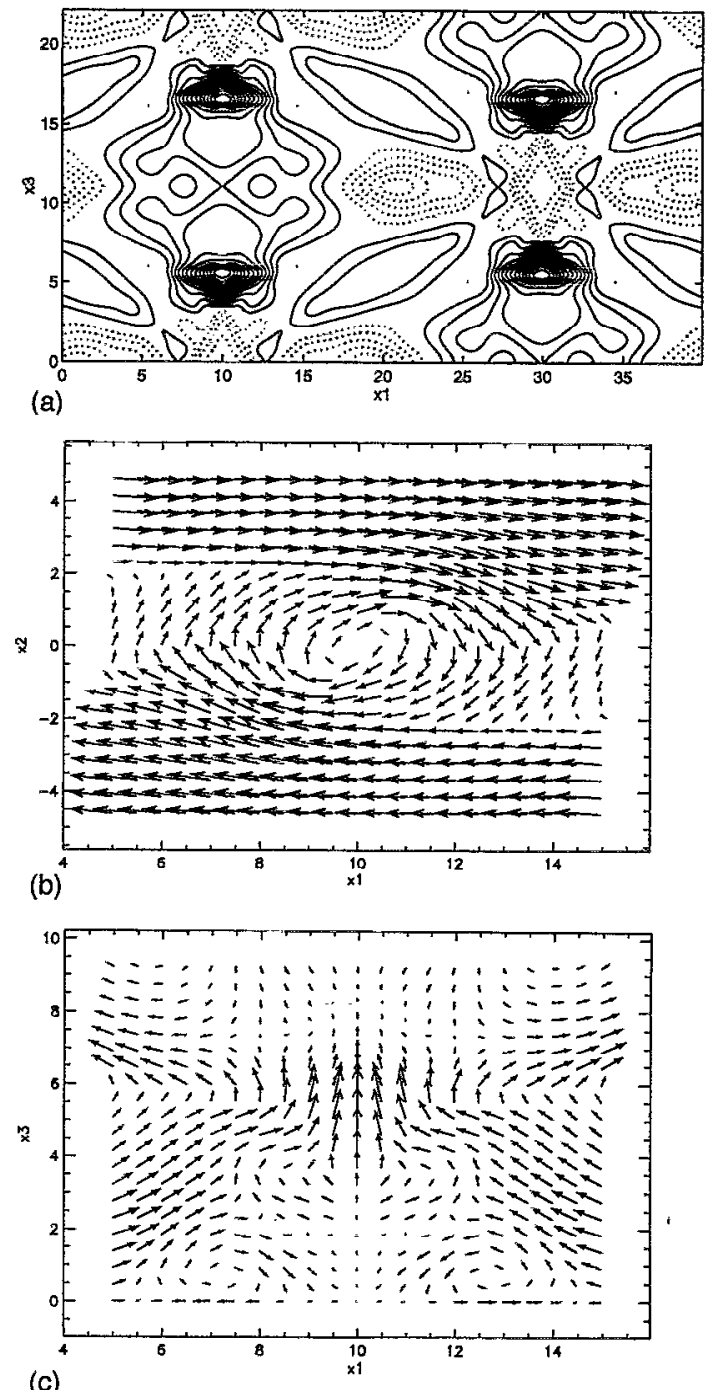

FIG. 1. Contours of the pressure at $t=122$ in the plane $x_{2}=0$ with $p<p_{\infty}$ (solid) and $p>p_{\infty}$ (dashed) with contour increment 0.025 (a). Velocity vectors at $t=122$ in the plane $x_{3}=5.5(\mathrm{~b})$ and in the plane $x_{2}=0(\mathrm{c})$. In order to reduce the density of the arrows, an arrow is plotted for only one in four grid points in each direction.

shock at the point $(10.0,0.0,6.0)$. Because the shock contains strong gradients in $x_{3}$, we say that its orientation is in the $x_{3}$ direction. If the fluid passes through a shock, the pressure jumps from a low to a high value. In general, low-pressure regions correspond to the cores of vortices, whereas highpressure regions are associated with stagnation points. The velocity vectors in Fig. 1(b) display a spanwise vortex in the plane $x_{3}=5.5$, which is in front of the shock. Figure $1(\mathrm{c})$ shows the velocity vectors in the plane $x_{2}=0$. The axis of the vortex is at $x_{1}=10.0$. The spanwise vortex in front of the shock accelerates fluid along its axis in the $x_{3}$ direction. The acceleration is so strong that the velocity attains supersonic values and, hence, a shock is formed through which the fluid passes in order to reach the stagnation point. Therefore, this shock is created by a sucking vortex that accelerates the fluid along its axis.

Next, we verify the shock jump relations that have been derived for stationary inviscid flow. The flow is nonstation-

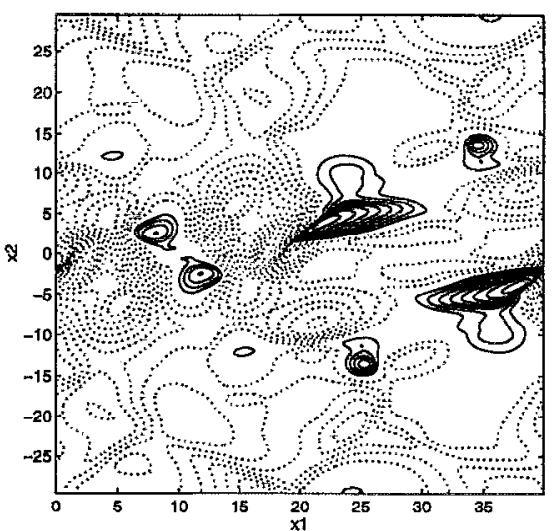

(a)

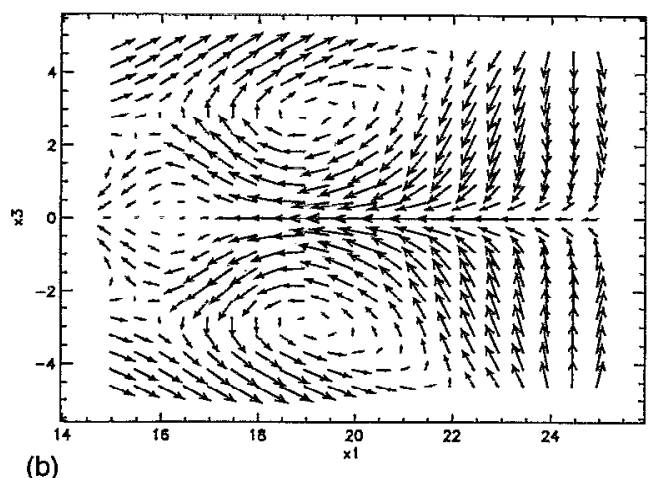

FIG. 2. Pressure at $t=182$ in the plane $x_{3}=0$ with $p<p_{\infty}$ (solid) and $p>p_{\infty}$ (dashed) with the contour increment is 0.025 (a). Velocity vectors at $t=182$ in the plane $x_{2}=1.6(\mathrm{~b})$.

ary, but the speed of the shock is negligibly small during the period in which the shock exists. The simulation data for this shock yields an upstream Mach number $M_{1}=1.40$ and the ratios $p_{2} / p_{1}=2.10$ and $\rho_{2} / \rho_{1}=1.67$, where the subscripts 1 and 2 refer to the up- and downstream values, respectively. The standard tables for normal shock waves give 2.12 and 1.69 for the pressure and density ratios corresponding to $M_{1}=1.40$. In viscous flow shocks have a finite thickness and in our case the shock thickness is approximately 0.35 , equal to three times the grid size.

The orientation, shape, and related vortex structure of the shocks at $t=182$ is different from $t=122$. Figure 2(a) shows the pressure in the planc $x_{3}=0$, which contains the maximum pressure gradient at this time. The two shocks in this plane are symmetric and a similar pattern is found in the plane $x_{3}=11.05$. From the structures considered, the shock at this time is most similar to the recent experimental visualization of a shock in a mixing layer. ${ }^{6}$ In order to study the flow structure around $(18.5,1.6,0.0)$ in more detail, we turn to the velocity vector plot in the plane $x_{2}=1.6$ [Fig. 2(b)]. Two counter-rotating vortices are observed. Not surprisingly, the velocity attains relatively high values in the region between these two vortices, and becomes supersonic. A shock wave occurs, since the velocity of the fluid has to be reduced in order to reach the stagnation point farther downstream. The mechanism that creates shocks is not unique; at $t=122$ the supersonic flow along the axis of a vortex creates a shock, whereas at $t=182$ the supersonic flow between a pair 


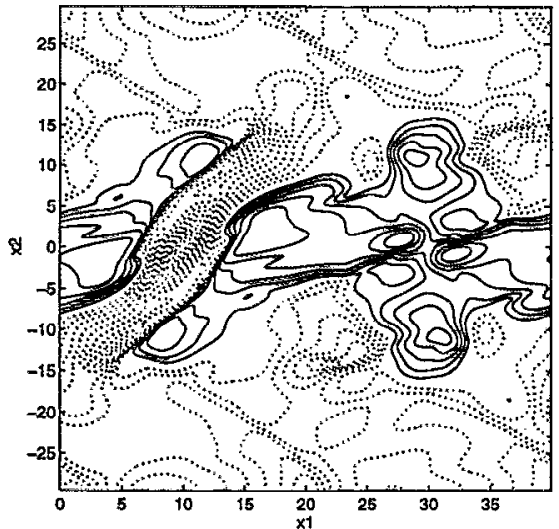

(a)

(b)

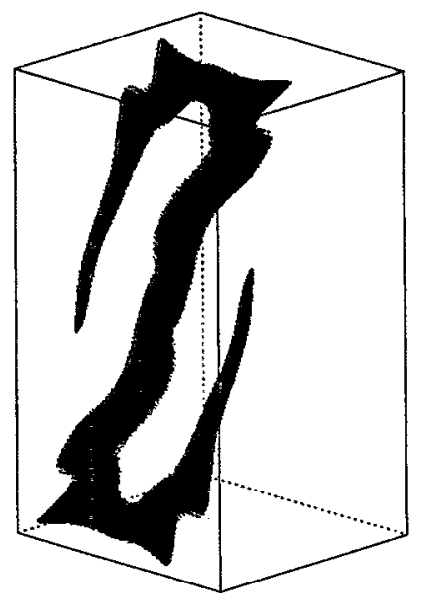

FIG. 3. Pressure at $t=200$ in the plane $x_{3}=3.9$ with $p<p_{\infty}$ (solid) and $p>p_{\infty}$ (dashed), where the contour increment is 0.025 (a). Surface $\nabla \cdot \mathbf{u}$ $=-0.5$, showing the three-dimensional shape of the shocks at $t=200$ in the part of the domain given by $2.5<x_{1}<17.5,-14.8<x_{2}<14.8$ and $-7.5<x_{3}<7.5$ (b).

of counter-rotating vortices gives rise to a shock.

Considerably larger shocks appear at $t=200$. Figure 3(a) shows the pressure in the plane with maximum pressure gradient, $x_{3}=3.9$. The region enclosed by the pair of shocks is a high-pressure region and contains a stagnation point. In this case the shocks fill a relatively large part of the domain, and it is difficult to identify separate vortex structures that create the shocks. Figure 3(b) shows two large shock blades with a horseshoe shape, centered around $(10,0,0)$. The same structure appears at another location, centered around $(30,0,11.05)$. The shocks at $t=122$ and $t=182$ persist only for a relatively short time. However, from $t=200$ the flow contains shocks for a much longer time, until $t=225$. During this period the structure shown in Fig. 3(b) changes and breaks down into several parts, while new shocks appear with stronger gradients in the spanwise direction.

Finally, we consider the effect of shocks on the turbulent dissipation rate in the Reynolds-averaged approach. The turbulent dissipation rate is defined as $\epsilon=\overline{\sigma_{i j} \partial u_{i}^{\prime \prime} / \partial x_{j}}$, where $\sigma_{i j}$ is the viscous stress tensor and the overbar and double prime denote the Reynolds average and Favre fluctuations, respectively. Sarkar et al. ${ }^{7}$ and Zeman ${ }^{8}$ separate the turbulent dissipation rate $\epsilon$ in a solenoidal part $\epsilon_{s}=\mu \overline{\omega_{i}^{\prime \prime} \omega_{i}^{\prime \prime}}$, where

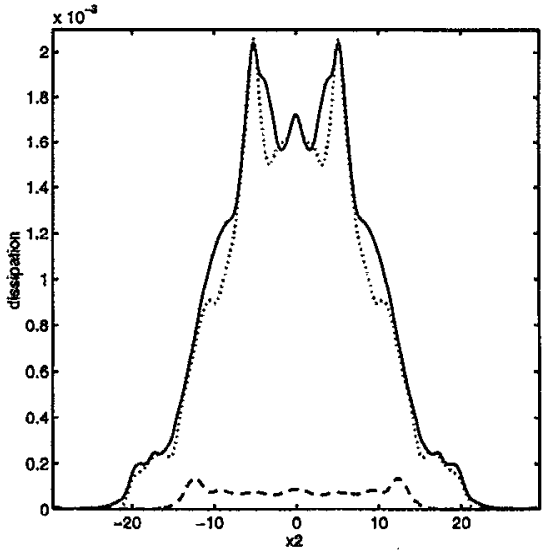

FIG. 4. Profiles of the turbulent dissipation rate $\epsilon$ (solid), $\epsilon_{s}$ (dotted), and $\epsilon_{d}$ (dashed) at $t=200$.

$\omega_{i}^{\prime \prime}$ denotes the fluctuating vorticity vector, and a dilatational part $\epsilon_{d}=\frac{4}{3} \mu \overline{\left(\nabla \cdot \mathbf{u}^{\prime \prime}\right)^{2}}$, which is a pure compressibility effect. For convenience of notation, we assume the viscosity $\mu$ to be constant. The decomposition $\epsilon=\epsilon_{s}+\epsilon_{d}$ is only valid in homogeneous turbulence, whereas in flows with one inhomogeneous direction $\left(x_{2}\right)$,

$$
\int \epsilon d x_{2}=\int \epsilon_{s} d x_{2}+\int \epsilon_{d} d x_{2} .
$$

Figure 4 shows the profiles of $\epsilon, \epsilon_{s}$, and $\epsilon_{d}$ at $t=200$, when the largest shocks occur. The dilatational fraction of the dissipation is less than $10 \%$ at this time and even smaller at all other times. When shocks are absent, the fraction is even less than $1 \%$. As a conclusion, the dilatational dissipation is increased by shocks, but it remains small compared to the total turbulent dissipation rate.

\section{ACKNOWLEDGMENTS}

The authors are grateful to Dr. N. D. Sandham and Dr. K. Luo (University of London) for stimulating discussions, and to Dr. J. W. van der Burg (NLR, Amsterdam) for his advice on the use of shock-capturing schemes. Furthermore, they thank NWO for providing computing time through NCF.

${ }^{1}$ S. K. Lele, "Compressibility effects on turbulence," Annu. Rev. Fluid Mech. 16, 211 (1994).

${ }^{2}$ N. D. Sandham and W. C. Reynolds, "Three-dimensional simulations of large eddies in the compressible mixing layer," J. Fluid Mech. 224, 133 (1991).

${ }^{3} \mathrm{~K}$. H. Luo and N. D. Sandham, "Mixing transition in compressible flow," Direct and Large-Eddy Simulation I, edited by P. R. Voke, L. Kleiser, and J. P. Chollet (Kluwer, New York, 1994), p. 335.

${ }^{4}$ A. W. Vreman, B. J. Geurts, and J. G. M. Kuerten, "Subgrid-modelling in LES of compressible flows," in Ref. 7, p. 133.

${ }^{5}$ J. W. van der Burg, "Numerical techniques for transonic flow calculations," Ph.D. dissertation, University of Twente, 1993.

${ }^{6} \mathrm{D}$. Papamoschou, "Evidence of shocklets in a counterflow supersonic shear layer," Phys. Fluids 7, 233 (1995).

${ }^{7}$ S. Sarkar, G. Erlebacher, M. Y. Hussaini, and H. O. Kreiss, "The analysis and modelling of dilatational terms in compressible turbulence," J. Fluid Mech. 225, 473 (1991).

${ }^{8} \mathrm{O}$. Zeman, "Dilatation dissipation: The concept and application in modeling compressible mixing layers," Phys. Fluids A 2, 178 (1990). 